National and Global Petroleum Assessment

\title{
Assessment of Undiscovered Conventional Oil and Gas Resources of China, 2020
}

Using a geology-based assessment methodology, the U.S. Geological Survey estimated undiscovered, technically recoverable mean resources of 13.4 billion barrels of oil and 244.4 trillion cubic feet of gas in nine geologic provinces of China.

\section{Introduction}

The U.S. Geological Survey (USGS) quantitatively assessed the potential for undiscovered, technically recoverable conventional oil and gas resources in nine geologic provinces of China (fig. 1). This assessment includes the Tarim Basin, Junggar Basin, Turpan Basin, Qaidam Basin, Sichuan Basin, Ordos Basin, Bohaiwan Basin, Songliao Basin, and the East China Sea Basin Provinces. Within these 9 provinces, 16 geologic assessment units (AUs) were defined, and each AU was assessed for undiscovered conventional oil, gas, and natural-gas liquids.

China contains a mosaic of cratonic terranes, remnants of oceanic crust, orogenic belts, suture zones, accretionary complexes, island-arc assemblages, and regional faults that record a complex history of terrane accretion and orogeny along the southern and eastern margins of Eurasia (Liu and others, 2013; Zheng and others, 2013; Zhao and others, 2014; Han and Zhao, 2018; Zhou and others, 2018). Beginning in the Paleozoic, several cratonic blocks separated diachronously from the northern margin of Gondwana and translated north across the Tethys Ocean as oceanic crust was subducted; these terranes eventually collided and accreted, knitting together a collage of tectonic elements. Major cratonic terranes that accreted to Eurasia included the Tarim Basin, Ordos Basin, and Sichuan Basin Provinces. In contrast, the basement of the Junggar, Turpan, Qaidam, and Songliao Basin Provinces are interpreted as fragments of oceanic crust that were not subducted, but rather were incorporated into orogenic belts (Mao and others, 2016; Han and Zhao, 2018). As accretion proceeded, the margins of the cratonic and oceanic fragments became sites of fold and thrust belts, suture zones, faults, and an amalgamation of island-arc and accretionary complexes; several of the terranes developed foreland basins. By the Permian, compressive deformation developed sufficient tectonic topography to isolate several of the basins from marine waters. This topographic relief led to hydraulically closed basins (Garcia-Castellanos, 2006; Marenssi and others, 2020), characterized by the development of extensive, basinwide lacustrine systems. The Junggar and Turpan Basin Provinces developed lacustrine systems by compressive deformation along the margins in the Permian, and lacustrine systems formed following compressional deformation in the Sichuan, Ordos, Tarim, and Qaidam Basin Provinces. In contrast, horst and graben systems along the eastern margin of Eurasia were formed by widespread back-arc extension related to changing motions of the Pacific plate. Extensive lacustrine systems formed within grabens in the Songliao, Bohaiwan, and East China Sea Basin Provinces (Li and others, 2012; Liang and Wang, 2019; Yang and others, 2020).

Petroleum source rocks within these nine provinces reflect the long and complex tectonic history (Jiang and others, 2016). As the cratonic blocks separated from Gondwana and traversed the Tethyan realm in the early Paleozoic, organic-rich marine sediments were deposited in basinal positions mainly associated with passive margin carbonate platforms, such as in the Tarim, Sichuan, and Ordos Basin Provinces (Yang and others, 2005). In the late Paleozoic, subsequent terrane collisions led to the development of marginal fold belts and associated foreland basins. Potential source rocks in the upper Paleozoic foreland basins are dominated by marginal marine to nonmarine, coal-bearing gas-prone sequences. Beginning in the Permian, along with the compressional formation of hydraulically closed basins, climatic conditions were appropriate to form extensive lacustrine systems with viable lacustrine source rocks, which are well known in the basins of China (Jiang and others, 2016).

The Sichuan Basin Province, which resides in the western part of the South China terrane, provides an example of the evolution of the tectonic control on the development of petroleum source rocks (Shi and others, 2016; Mu and others, 2019). The cratonic terrane of the Sichuan Basin Province was rimmed by passive margins as the terrane

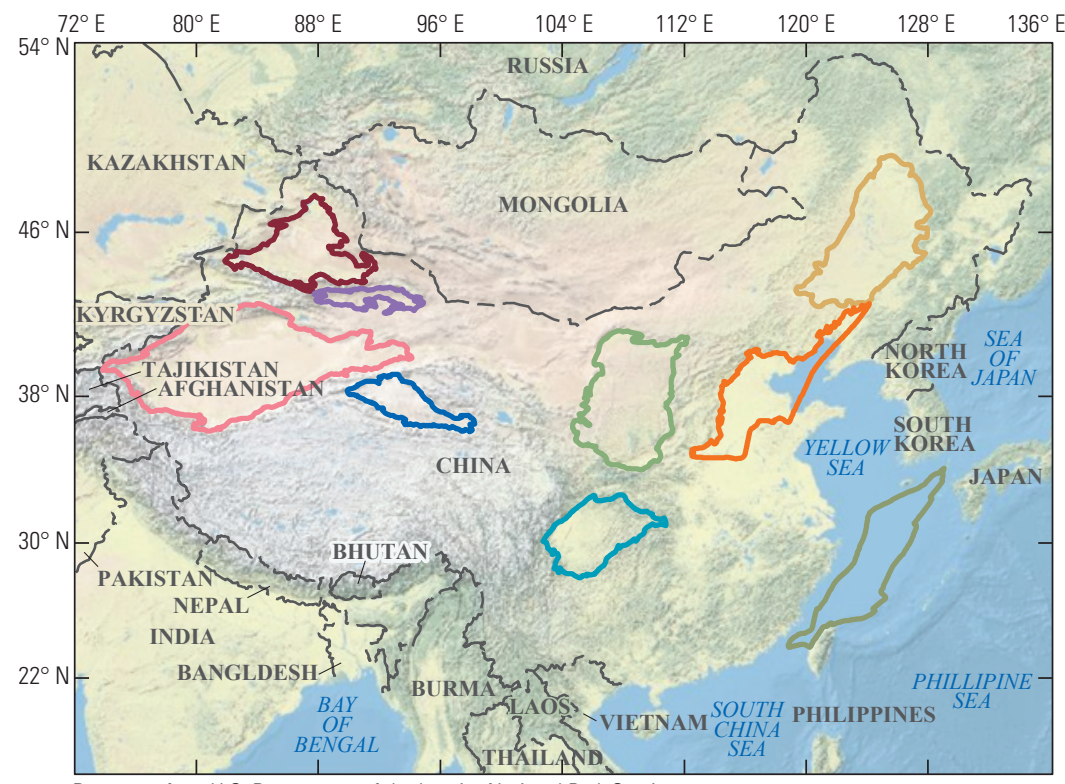

Base map from U.S. Department of the Interior National Park Service

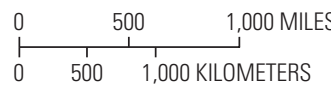

\section{EXPLANATION}

Tarim Basin Province Junggar Basin Province

Turpan Basin Province

Qaidam Basin Province

Sichuan Basin Province

Ordos Basin Province

Bohaiwan Basin Province

Songliao Basin Province

East China Sea Basin Province

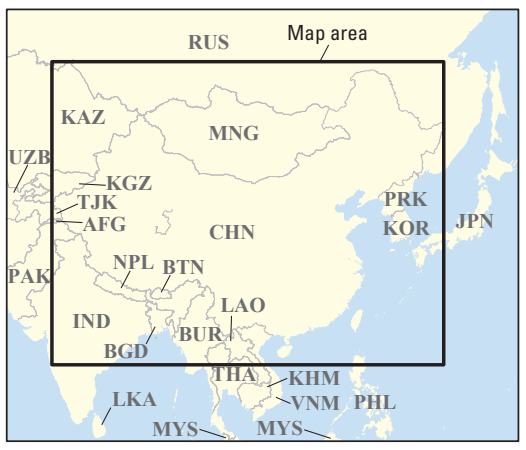

Figure 1. Map showing the location of nine geologic provinces of China assessed in this study. 
moved north from Gondwana, which led to the deposition of widespread organic-rich marine source rocks. As the South China terrane impinged on the Eurasia margin, a foreland basin was formed in the Sichuan Basin Province and was the depositional site for marine, marginal marine, and coal-bearing sequences. In the Late Triassic, as the South China terrane collided with the North China and Songpan Ganzi terranes, compressional deformation resulted in topography, a hydraulically closed basin, and the development of an extensive basinwide lacustrine system. A similar temporal development of marine to nonmarine source rocks is interpreted for the Tarim Basin Province (Jin and others, 2008; Wang and others, 2019). In contrast, because the Junggar Basin Province is floored by upper Paleozoic oceanic crust, there is no lower Paleozoic section. Permian deformation isolated the Junggar Basin from marine waters, and the principal petroleum source rocks developed within a lacustrine depositional system. The Turpan and Qaidam Basin Provinces illustrate a similar tectonic development of lacustrine source-rock conditions.

The Bohaiwan Basin Province is an example of the development of lacustrine conditions in an extensional system resulting from complex motion during the subduction of the Pacific plate (Allen and others, 1998). Extension resulted in a regional system of horsts and grabens, which were isolated from marine waters, and many of the grabens developed lacustrine depositional systems (Feng and others, 2016). This process also led to lacustrine systems being formed in the Songliao and East China Sea Basin Provinces (Ge and others, 2012; Wang and others, 2016; Liang and Wang, 2019; Yang and others, 2020). Key assessment input data are summarized in table 1 .

Table 1. Key input data for 16 conventional assessment units in China.

[Shading indicates not applicable. AU, assessment unit; $\mathrm{MMBO}$, million barrels of oil; BCFG, billion cubic feet of gas]

\begin{tabular}{|c|c|c|c|c|c|c|c|c|}
\hline \multirow{2}{*}{$\begin{array}{c}\text { Assessment input data- } \\
\text { Conventional AUs }\end{array}$} & \multicolumn{4}{|c|}{ Tarim Mesozoic-Cenozoic Reservoirs AU } & \multicolumn{4}{|c|}{ Tarim Paleozoic Reservoirs AU } \\
\hline & Minimum & Median & Maximum & Calculated mean & Minimum & Median & Maximum & Calculated mean \\
\hline Number of oil fields & 1 & 30 & 90 & 31.9 & 1 & 100 & 200 & 102.5 \\
\hline Number of gas fields & 1 & 50 & 150 & 53.2 & 1 & 250 & 500 & 256.1 \\
\hline Size of oil fields (MMBO) & 5 & 8 & 200 & 12.2 & 5 & 8 & 3,500 & 37.3 \\
\hline Size of gas fields (BCFG) & 30 & 60 & 20,000 & 260.1 & 30 & 60 & 20,000 & 260.1 \\
\hline AU probability & 1.0 & & & & 1.0 & & & \\
\hline \multirow{2}{*}{$\begin{array}{l}\text { Assessment input data- } \\
\text { Conventional AUs }\end{array}$} & \multicolumn{4}{|c|}{ Junggar Upper Paleozoic-Cenozoic Reservoirs AU } & \multicolumn{4}{|c|}{ Turpan Mesozoic-Cenozoic Reservoirs AU } \\
\hline & Minimum & Median & Maximum & Calculated mean & Minimum & Median & Maximum & Calculated mean \\
\hline Number of oil fields & 1 & 45 & 90 & 46.1 & 1 & 12 & 25 & 12.3 \\
\hline Number of gas fields & 1 & 80 & 160 & 82.0 & 1 & 12 & 25 & 12.3 \\
\hline Size of oil fields (MMBO) & 5 & 10 & 700 & 21.7 & 5 & 8 & 200 & 12.2 \\
\hline Size of gas fields (BCFG) & 30 & 72 & 12,000 & 230.8 & 30 & 60 & 3,500 & 122.0 \\
\hline AU probability & 1.0 & & & & 1.0 & & & \\
\hline \multirow{2}{*}{$\begin{array}{c}\text { Assessment input data- } \\
\text { Conventional AUs }\end{array}$} & \multicolumn{4}{|c|}{ Oaidam Mesozoic-Cenozoic Reservoirs AU } & \multicolumn{4}{|c|}{ Sichuan Southeast Fold Belt Reservoirs AU } \\
\hline & Minimum & Median & Maximum & Calculated mean & Minimum & Median & Maximum & Calculated mean \\
\hline Number of oil fields & 1 & 40 & 80 & 41.0 & & & & \\
\hline Number of gas fields & 1 & 40 & 80 & 41.0 & 1 & 300 & 600 & 307.3 \\
\hline Size of oil fields (MMBO) & 5 & 10 & 200 & 14.8 & & & & \\
\hline Size of gas fields (BCFG) & 30 & 72 & 5,000 & 160.2 & 6 & 36 & 10,000 & 161.9 \\
\hline AU probability & 1.0 & & & & 1.0 & & & \\
\hline \multirow{2}{*}{$\begin{array}{c}\text { Assessment input data- } \\
\text { Conventional AUs }\end{array}$} & \multicolumn{4}{|c|}{ Sichuan Foreland Basin Reservoirs AU } & \multicolumn{4}{|c|}{ Sichuan Central Uplift Reservoirs AU } \\
\hline & Minimum & Median & Maximum & Calculated mean & Minimum & Median & Maximum & Calculated mean \\
\hline Number of oil fields & & & & & 1 & 4 & 8 & 4.1 \\
\hline Number of gas fields & 1 & 150 & 300 & 153.7 & 1 & 8 & 16 & 8.2 \\
\hline Size of oil fields (MMBO) & & & & & 1 & 2 & 20 & 2.6 \\
\hline Size of gas fields (BCFG) & 6 & 36 & 3,000 & 92.1 & 6 & 12 & 100 & 14.8 \\
\hline AU probability & 1.0 & & & & 1.0 & & & \\
\hline \multirow{2}{*}{$\begin{array}{c}\text { Assessment input data- } \\
\text { Conventional AUs }\end{array}$} & \multicolumn{4}{|c|}{ Ordos Lower Paleozoic Conventional Reservoirs AU } & \multicolumn{4}{|c|}{ Ordos Western Deformed Belt Reservoirs AU } \\
\hline & Minimum & Median & Maximum & Calculated mean & Minimum & Median & Maximum & Calculated mean \\
\hline Number of oil fields & & & & & 1 & 10 & 30 & 10.6 \\
\hline Number of gas fields & 1 & 50 & 150 & 53.2 & 1 & 30 & 90 & 31.9 \\
\hline Size of oil fields (MMBO) & & & & & 5 & 8 & 200 & 12.2 \\
\hline Size of gas fields (BCFG) & 30 & 48 & 6,000 & 123.3 & 30 & 72 & 10,000 & 212.6 \\
\hline AU probability & 1.0 & & & & 0.9 & & & \\
\hline \multirow{2}{*}{$\begin{array}{l}\text { Assessment input data- } \\
\text { Conventional AUs }\end{array}$} & \multicolumn{4}{|c|}{ Ordos Mesozoic Sandstone Reservoirs AU } & & Bohaiwan & ozoic Resen & AU \\
\hline & Minimum & Median & Maximum & Calculated mean & Minimum & Median & Maximum & Calculated mean \\
\hline Number of oil fields & 1 & 40 & 80 & 41.0 & 1 & 500 & 1,000 & 512.2 \\
\hline Number of gas fields & & & & & 1 & 70 & 210 & 74.4 \\
\hline Size of oil fields (MMBO) & 5 & 8 & 400 & 14.8 & 1 & 2 & 600 & 8.2 \\
\hline Size of gas fields (BCFG) & & & & & 6 & 24 & 2,500 & 66.0 \\
\hline AU probability & 1.0 & & & & 1.0 & & & \\
\hline Assessment input data- & Bohaiv & Mid-Prote & jic-Paleozoi & eservoirs AU & & ggliao Syn & Sourced Res & jirs AU \\
\hline Conventional AUs & Minimum & Median & Maximum & Calculated mean & Minimum & Median & Maximum & Calculated mean \\
\hline Number of oil fields & 1 & 150 & 450 & 159.5 & 1 & 10 & 20 & 10.3 \\
\hline Number of gas fields & 1 & 50 & 150 & 53.2 & 1 & 50 & 100 & 51.2 \\
\hline Size of oil fields (MMBO) & 1 & 2 & 40 & 3.0 & 5 & 8 & 200 & 12.2 \\
\hline Size of gas fields (BCFG) & 6 & 24 & 3,000 & 71.5 & 30 & 72 & 7,000 & 182.7 \\
\hline AU probability & 1.0 & & & & 1.0 & & & \\
\hline Assessment input data- & & liao Post-R & Sourced Res & oirs AU & & East Ch & ea Reservoi & \\
\hline Conventional AUs & Minimum & Median & Maximum & Calculated mean & Minimum & Median & Maximum & Calculated mean \\
\hline Number of oil fields & 1 & 80 & 160 & 82.0 & 1 & 30 & 90 & 32.0 \\
\hline Number of gas fields & 1 & 10 & 20 & 10.3 & 1 & 80 & 240 & 85.1 \\
\hline Size of oil fields (MMBO) & 5 & 8 & 300 & 13.6 & 5 & 10 & 1,000 & 24.9 \\
\hline Size of gas fields (BCFG) & 30 & 72 & 4,000 & 147.7 & 30 & 72 & 12,000 & 230.8 \\
\hline AU Probability & 1.0 & & & & 1.0 & & & \\
\hline
\end{tabular}




\section{Undiscovered Resources Summary}

The USGS quantitatively assessed undiscovered oil, gas, and natural gas liquids resources within nine geologic provinces of China (table 2). The fully risked mean totals are 13,400 million barrels of oil (MMBO) or 13.4 billion barrels of oil, with a F95-F5 fractile range from 7,022 to 22,267 MMBO; 244,436 billion cubic feet of gas
(BCFG), or 244.4 trillion cubic feet, with an F95-F5 range from 122,343 to $412,088 \mathrm{BCFG}$; and 3,775 million barrels of natural gas liquids (MMBNGL), or 3.8 billion barrels of natural gas liquids, with an F95-F5 range from 1,819 to 6,561 MMBNGL. Assessment results were published previously for the Pearl River Mouth Basin, Beibuwan Basin, Qiongdongnan Basin, and Taixinan Basin Provinces (Schenk and others, 2020).

Table 2. Results for 16 conventional assessment units in China.

[Results shown are fully risked estimates. F95 represents a 95-percent chance of at least the amount tabulated; other fractiles are defined similarly. Shading indicates not applicable. MMBO, million barrels of oil; BCFG, billion cubic feet of gas; NGL, natural gas liquids; MMBNGL, million barrels of natural gas liquids]

\begin{tabular}{|c|c|c|c|c|c|c|c|c|c|c|c|c|c|c|}
\hline \multirow{3}{*}{$\begin{array}{l}\text { Total petroleum system and } \\
\text { assessment units (AUs) }\end{array}$} & \multirow{3}{*}{$\begin{array}{c}\text { AU } \\
\text { probability }\end{array}$} & \multirow{3}{*}{$\begin{array}{c}\text { Accumulation } \\
\text { type }\end{array}$} & \multicolumn{12}{|c|}{ Total undiscovered resources } \\
\hline & & & \multicolumn{4}{|c|}{ Oil (ММво) } & \multicolumn{4}{|c|}{ Gas (BCFG) } & \multicolumn{4}{|c|}{ NGL (MMBNGL) } \\
\hline & & & F95 & F50 & F5 & Mean & F95 & F50 & F5 & Mean & F95 & F50 & F5 & Mean \\
\hline \multicolumn{15}{|c|}{ Mesozoic Composite Total Petroleum System, Tarim Basin Province } \\
\hline \multirow{2}{*}{$\begin{array}{l}\text { Tarim Mesozoic_Cenozoic } \\
\text { Reservoirs AU }\end{array}$} & \multirow{2}{*}{1.0} & Oil & 181 & 361 & 687 & 388 & 380 & 758 & 1,441 & 815 & 12 & 24 & 46 & 26 \\
\hline & & Gas & & & & & 4,318 & 12,037 & 29,875 & 13,925 & 156 & 433 & 1,075 & 501 \\
\hline \multicolumn{15}{|c|}{ Lower Paleozoic Composite Total Petroleum System, Tarim Basin Province } \\
\hline \multirow{2}{*}{ Tarim Paleozoic Reservoirs AU } & \multirow{2}{*}{1.0} & Oil & 1,680 & 3,505 & 7,013 & 3,820 & 3,862 & 8,064 & 16,123 & 8,787 & 89 & 185 & 371 & 202 \\
\hline & & Gas & & & & & 37,067 & 64,208 & 103,939 & 66,508 & 1,001 & 1,731 & 2,807 & 1,796 \\
\hline \multicolumn{15}{|c|}{ Paleozoic-Mesozoic Composite Total Petroleum System, Junngar Basin Province } \\
\hline \multirow{2}{*}{\begin{tabular}{|c|} 
Junggar Upper Paleozoic- \\
Cenozoic Reservoirs AU
\end{tabular}} & \multirow{2}{*}{1.0} & Oil & 525 & 947 & 1,657 & 1,000 & 469 & 852 & 1,498 & 900 & 6 & 10 & 18 & 11 \\
\hline & & Gas & & & & & 9,641 & 17,877 & 31,791 & 18,949 & 115 & 214 & 383 & 227 \\
\hline \multicolumn{15}{|c|}{ Paleozoic-Mesozoic Composite Total Petroleum System, Turpan Basin Province } \\
\hline \multirow{2}{*}{$\begin{array}{l}\text { Turpan Mesozoic-Cenozoic } \\
\text { Reservoirs AU }\end{array}$} & \multirow{2}{*}{1.0} & Oil & 77 & 139 & 262 & 150 & 77 & 139 & 263 & 150 & 2 & 3 & 6 & 3 \\
\hline & & Gas & & & & & 612 & 1,307 & 3,101 & 1,507 & 15 & 33 & 78 & 38 \\
\hline \multicolumn{15}{|c|}{ Mesozoic-Cenozoic Composite Total Petroleum System, Qaidam Basin Province } \\
\hline Qaidam Mesozoic-Cenozoic & 10 & Oil & 368 & 589 & 906 & 606 & 587 & 941 & 1,451 & 970 & 1 & 1 & 2 & 1 \\
\hline Reservoirs AU & 1.0 & Gas & & & & & 3,350 & 6,170 & 11,011 & 6,547 & 12 & 22 & 39 & 23 \\
\hline & & aleozoic-Mesoz & oic Com & osite To & al Petro & eum Sys & em, Sichu & $n B \operatorname{asin} P$ & rovince & & & & & \\
\hline $\begin{array}{l}\text { Sichuan Southeast Fold Belt } \\
\text { Reservoirs AU }\end{array}$ & 1.0 & Gas & & & & & 29,376 & 48,300 & 75,259 & 49,808 & 41 & 72 & 118 & 75 \\
\hline $\begin{array}{l}\text { Sichuan Foreland Basin } \\
\text { Reservoirs AU }\end{array}$ & 1.0 & Gas & & & & & 8,500 & 13,744 & 21,184 & 14,142 & 16 & 26 & 42 & 27 \\
\hline Sichuan Central Uplift & 10 & Oil & 5 & 10 & 19 & 10 & 8 & 15 & 28 & 16 & 0 & 0 & 0 & 0 \\
\hline Reservoirs AU & & Gas & & & & & 70 & 116 & 191 & 122 & 1 & 2 & 3 & 2 \\
\hline & & Paleozoic C & omposi & e Total P & etroleum & System, & Drdos Bas & $n$ Provinc & & & & & & \\
\hline $\begin{array}{l}\text { Ordos Lower Paleozoic } \\
\quad \text { Conventional Reservoirs AU }\end{array}$ & 1.0 & Gas & & & & & 2,571 & 5,960 & 12,647 & 6,558 & 2 & 5 & 12 & 6 \\
\hline Ordos Western Deformed Belt & 09 & Oil & 0 & 110 & 246 & 117 & 0 & 22 & 50 & 23 & 0 & 0 & 0 & 0 \\
\hline Reservoirs AU & 0.3 & Gas & & & & & 0 & 5,457 & 13,928 & 6,097 & 0 & 5 & 13 & 5 \\
\hline & & Cenozoic C & omposi & Total P & etroleum & System, & Ordos Bas & Provinc & & & & & & \\
\hline $\begin{array}{l}\text { Ordos Mesozoic Sandstone } \\
\text { Reservoirs AU }\end{array}$ & 1.0 & Oil & 334 & 576 & 976 & 605 & 65 & 115 & 199 & 121 & 0 & 0 & 0 & 0 \\
\hline & & Cenozoic Cor & nposite & otal Pet & oleum S & stem, $B c$ & haiwan B & sin Provin & & & & & & \\
\hline Bohaiwan Cenozoic & 10 & Oil & 2,576 & 4,093 & 6,234 & 4,203 & 2,557 & 4,086 & 6,260 & 4,203 & 20 & 33 & 50 & 34 \\
\hline Reservoirs AU & & Gas & & & & & 2,055 & 4,550 & 8,934 & 4,896 & 56 & 123 & 241 & 132 \\
\hline Bohaiwan Mid-Proterozoic- & 10 & Oil & 241 & 444 & 798 & 471 & 216 & 399 & 720 & 424 & 5 & 8 & 15 & 9 \\
\hline Paleozoic Reservoirs AU & & Gas & & & & & 1,456 & 3,472 & 7,309 & 3,802 & 34 & 80 & 168 & 87 \\
\hline & & Songliao Syn-Rif & $\mathrm{tCompc}$ & site Tota & Petrole & m Syster & , Songlia & Basin $\mathrm{Pr}$ & ovince & & & & & \\
\hline Songliao Syn-Rift Sourced & 10 & Oil & 64 & 114 & 222 & 125 & 166 & 297 & 577 & 324 & 1 & 2 & 4 & 2 \\
\hline Reservoirs AU & & Gas & & & & & 4,704 & 8,798 & 15,880 & 9,350 & 42 & 79 & 144 & 84 \\
\hline Songliao Post-Rift Sourced & 10 & Oil & 682 & 1,082 & 1,661 & 1,114 & 403 & 648 & 1,008 & 669 & 1 & 1 & 2 & 1 \\
\hline Reservoirs AU & & Gas & & & & & 583 & 1,293 & 3,228 & 1,514 & 12 & 27 & 68 & 32 \\
\hline & Mes & ozoic-Cenozoic & Compos & te Total & etroleun & System, & East Chin & Sea Basi & in Provinc & & & & & \\
\hline East China Sea Reservoirs AU & 1.0 & Oil & 289 & 703 & 1,586 & 791 & 1,331 & 3,234 & 7,301 & 3,640 & 21 & 52 & 117 & 58 \\
\hline & 1.0 & Gas & & & & & 7,919 & 18,120 & 36,892 & 19,669 & 158 & 363 & 739 & 393 \\
\hline $\begin{array}{l}\text { Total undiscovered } \\
\text { conventional resources }\end{array}$ & & & 7,022 & 12,673 & 22,267 & 13,400 & 122,343 & 230,979 & 412,088 & 244,436 & 1,819 & 3,534 & 6,561 & 3,775 \\
\hline
\end{tabular}




\section{References Cited}

Allen, M.B., Macdonald, D.I.M., Xun, Z., Vincent, S.J., and BronetMenzies, C., 1998, Transtensional deformation in the evolution of the Bohai Basin, northern China, in Holdsworth, R.E., Strachan, R.S., and Dewey, J.F., eds., Continental transpressional and transtensional tectonics: The Geological Society of London Special Publication 135, p. 215-229.

Feng, Y., Jiang, S., Hu, S., Li, S., Lin, C., and Xie, X., 2016, Sequence stratigraphy and importance of syndepositional structural slope-break for architecture of Paleogene syn-rift lacustrine strata, Bohai Bay Basin, China: Marine and Petroleum Geology, v. 69, p. 183-204.

Garcia-Castellanos, D., 2006, Long-term evolution of tectonic lakesClimatic controls on the development of internally drained basins, in Willett, S.D., Hovius, N., Brandon, M.T., and Fisher, D.M., eds., Tectonics, climate, and landscape evolution: Geological Society of America Special Publication 398, p. 283-294. [Also available at https://doi.org/10.1130/2006.2398(17).]

Ge, R., Zhang, Q., Wang, L., Chen, J., Xie, G., and Wang, X., 2012, Late Mesozoic rift evolution and crustal extension in the central Songliao Basin, northeastern China-Constraints from cross-section restoration and implications for lithospheric thinning: International Geology Review, v. 54, no. 2, p. 183-207. [Also available at https://doi.org/10.1080/00206814.2010.511900.]

Han, Y., and Zhao, G., 2018, Final amalgamation of the Tianshan and Junggar orogenic collage in the southwestern Central Asian Orogenic Belt - Constraints on the closure of the Paleo-Asian ocean: EarthScience Reviews, v. 186, p. 129-152. [Also available at https://doi.org/10.1016/j.earscirev.2017.09.012.]

Jiang, S., Xu, Z., Feng, Y., Zhang, J., Cai, D., Chen, L., Wu, Y., Zhou, D., Bao, S., and Long, S., 2016, Geologic characteristics of hydrocarbon-bearing marine, transitional and lacustrine shales in China: Journal of Asian Earth Sciences, v. 115, p. 404-418. [Also available at https://doi.org/10.1016/j.jseaes.2015.10.016.]

Jin, Z., Yang, M., Lu, X., Sun, D., Tang, X., Peng, G., and Lei, G., 2008, The tectonics and petroleum system of the Qiultagh fold and thrust belt, northern Tarim Basin, NW China: Marine and Petroleum Geology, v. 25, no. 8, p. 767-777. [Also available at https://doi.org/ 10.1016/j.marpetgeo.2008.01.011.]

Li, S., Zhao, G., Dai, L., Liu, X., Zhou, L., Santosh, M., and Suo, Y., 2012, Mesozoic basins in eastern China and their bearing on the deconstruction of the North China craton: Journal of Asian Earth Sciences, v. 47, p. 64-79. [Also available at https://doi.org/10.1016/ j.jseaes.2011.06.008.]

Liang, J., and Wang, H., 2019, Cenozoic tectonic evolution of the East China Sea Shelf Basin and its coupling relationships with Pacific plate subduction: Journal of Asian Earth Sciences, v. 171, p. 376-387. [Also available at https://doi.org/10.1016/j.jseaes.2018.08.030.]

Liu, S., Su, S., and Zhang, G., 2013, Early Mesozoic basin development in North China-Indications of cratonic deformation: Journal of Asian Earth Sciences, v. 62, p. 221-236. [Also available at https://doi.org/10.1016/j.jseaes.2012.09.011.]
Mao, L., Xiao, A., Zhang, H., Wu, Z., Wang, L., Shen, Y., and Wu, L., 2016, Structural deformation patterns within the NW Qaidam Basin in the Cenozoic era and its tectonic implications: Tectonophysics 687, p. 78-93. [Also available at https://doi.org/10.1016/ j.tecto.2016.09.008.]

Marenssi, S.A., Limarino, C.O., Schencman, L.J., and Ciccioli, P.I., 2020, Tectonic and geomorphic controls on the lacustrine deposits of the Neogene Vinchina Basin, northwestern Argentina: Journal of Sedimentary Research, v. 90, no. 2, p. 250-267. [Also available at https://doi.org/10.2110/jsr.2020.13.]

Mu, H., Yan, D.-P., Qiu, L., Yang, W.-X., Kong, R.-Y., Gong, L.-X., and Li, S., 2019, Formation of the Late Triassic western Sichuan foreland basin of the Qinling Orogenic Belt, SW China-Sedimentary and geochronological constraints from the Xujiahe Formation: Journal of Asian Earth Sciences, v. 183, 20 p.

Schenk, C.J., Mercier, T.J., Woodall, C.A., Finn, T.M., Le, P.A., Marra, K.R., Leathers-Miller, H.M., and Drake, R.M., II, 2020, Assessment of undiscovered conventional oil and gas resources of Southeast Asia, 2020: U.S. Geological Survey Fact Sheet 2020-3046, 2 p. [Also available at https://doi.org/10.3133/fs20203046.]

Shi, H., Shi, X., Glasmacher, U.A., Yang, X., and Stockli, D.F., 2016, The evolution of the eastern Sichuan Basin, Yangtze block since Cretaceous - Constraints from low temperature thermochronology: Journal of Asian Earth Sciences, v. 116, p. 208-221. [Also available at https://doi.org/10.1016/j.jseaes.2015.11.008.]

Wang, P.-J., Mattern, F., Didenko, N.A., Zhu, D.-F., Singer, B., and Sun, X.-M., 2016, Tectonics and cycle system of the Cretaceous Songliao Basin-An inverted active continental margin basin: Earth-Science Reviews, v. 159, p. 82-102. [Also available at https://doi.org/10.1016/j.earscirev.2016.05.004.]

Wang, T., Jin, Z., Shi, Z., Dai, X., and Cheng, R., 2019, Phanerozoic plate history and structural evolution of the Tarim Basin, northwestern China: International Geology Review, v. 62, no. 12, p. 1555-1569. [Also available at https://doi.org/10.1080/00206814.2019.1661038.]

Yang, C., Sun, J., Yang, Y., Yang, C., and Wang, J., 2020, Key factors controlling Mesozoic hydrocarbon accumulation in the southern East China Sea Basin: Marine and Petroleum Geology, v. 118, 20 p.

Yang, Y., Li, W., and Ma, L., 2005, Tectonic and stratigraphic controls on hydrocarbon systems in the Ordos Basin-A multicycle cratonic basin in central China: American Association of Petroleum Geologists Bulletin, v. 89, no. 2, p. 255-269. [Also available at https://doi.org/10.1306/ 10070404027.]

Zhao, S., Li, S., Liu, X., Suo, Y., Dai, L., Lou, D., Sun, W., Li, T., Wang, X., and Yang, Z., 2014, Intracontinental orogenic transition-Insights from structures of the east Junggar Basin between the Altay and Tianshan orogens: Journal of Asian Earth Sciences, v. 88, p. 137-148. [Also available at https://doi.org/10.1016/j.jseaes.2014.03.008.]

Zheng, Y.-F., Xiao, W.-J., and Zhao, G., 2013, Introduction to tectonics of China: Gondwana Research, v. 23, no. 4, p. 1189-1206. [Also available at https://doi.org/10.1016/j.gr.2012.10.001.]

Zhou, J.-B., Wilde, S.A., Zhao, G.-C., and Han, J., 2018, Nature and assembly of microcontinental blocks within the Paleo-Asian ocean: Earth-Science Reviews, v. 186, p. 76-93. [Also available at https://doi.org/10.1016/j.earscirev.2017.01.012.]

\section{For More Information}

Assessment results are also available at the USGS Energy Resources Program website, https://www.usgs.gov/ energy-and-minerals/energy-resources-program.

\section{China Assessment Team}

Christopher J. Schenk, Tracey J. Mercier, Cheryl A. Woodall, Geoffrey S. Ellis, Thomas M. Finn, Phuong A. Le, Kristen R. Marra, Heidi M. Leathers-Miller, and Ronald M. Drake II. 